(C) 2017 IEEE. Personal use of this material is permitted. Permission from IEEE must be obtained for all other uses, in any current or future media, including reprinting/republishing this material for advertising or promotional purposes, creating new collective works, for resale or redistribution to servers or lists, or reuse of any copyrighted component of this work in other works. 


\title{
Inherent Fuzzy Entropy for the Improvement of EEG Complexity Evaluation
}

\author{
Zehong Cao, Chin-Teng Lin, Fellow, IEEE
}

\begin{abstract}
In recent years, the concept of entropy has been widely used to measure the dynamic complexity of signals. Since the state of complexity of human beings is significantly affected by their health state, developing accurate complexity evaluation algorithms is a crucial and urgent area of study. This paper proposes using inherent fuzzy entropy (Inherent FuzzyEn) and its multi-scale version, which employs empirical mode decomposition (EMD) and fuzzy membership function (exponential function) to address the dynamic complexity in electroencephalogram (EEG) data. In the literature, the reliability of entropy-based complexity evaluations has been limited by superimposed trends in signals and a lack of multiple time scales. Our proposed method represents the first attempt to use the inherent fuzzy entropy algorithm to increase the reliability of complexity evaluation in realistic EEG applications. We recorded the EEG signals of several subjects under resting condition, and the EEG complexity was evaluated using approximate entropy (ApEn), sample entropy (SampEn), fuzzy entropy (FuzzyEn) and Inherent FuzzyEn, respectively. The results indicate that Inherent FuzzyEn is superior to other competing models regardless of the use of fuzzy or non-fuzzy structures, and has the most stable complexity and smallest root mean square deviation (RMSD).
\end{abstract}

Index Terms - Complexity, EMD, Fuzzy, Entropy, EEG

\section{INTRODUCTION}

I recent times, numerous techniques have been rapidly developed for measuring dynamic complexity [1]. The notion of complexity is not precisely delineated, yet time series of dynamic complexity have been investigated via several measures, e.g., approximate entropy [2], sample entropy [3, 4] and fuzzy entropy [5]. Approximate entropy (ApEn) was presented as a measure of complexity in short and noisy recordings [2]. Sample entropy (SampEn) [4] was presented as

Manuscript received July 14, 2016; revised November 03, 2016; accepted January 18, 2017. This work was supported in part by Computational Intelligence and Brain Computer Interface (CI\&BCI) Centre, University of Technology Sydney, Australia, the Australian Research Council (ARC) under discovery grant DP150101645, in part by the Aiming for the Top University Plan of National Chiao Tung University, the Ministry of Education, Taiwan, under Contract 104W963. Research was also sponsored in part by the Army Research Laboratory and was accomplished under Cooperative Agreement Number W911NF-10-2-0022.

Z. Cao and C.T. Lin are with the Faculty of Engineering and Information Technology, University of Technology Sydney, Sydney, Australia, and also with the Department of Electrical and Computer Engineering and the Brain Research Center, National Chiao Tung University, Hsinchu, Taiwan (e-mail: zhcaonctu@gmail.com; Chin-Teng.Lin@uts.edu.au). having the advantage of being less dependent on the time series length. Multiscale sample entropy (MSE) [3] was presented as taking into account multiple time scales. Since MSE relies on the computation of the sample entropy over a range of scales, coarse-grained time series that represent the system dynamics on different scales are analyzed using the sample entropy algorithm. MSE has been used in different fields successfully, and is therefore a potential algorithm to quantify the complexity of signals.

Some state-of-the-art fuzzy theory based algorithms have been widely used in implementation of physiological signals processing [6-9]. To further address reliable complexity, the concept of fuzzy sets [10] was proposed to investigate the fuzzy entropy (FuzzyEn) measure [5, 11], which relies on fuzzy membership functions (smooth and continuous boundary) instead of the Heaviside function (hard and discontinuous boundary). Since fuzzy entropy measures a fuzzy boundary, it corresponds to a stronger relative reliability, therefore provides a more accurate complexity than sample entropy [11]. From previous theoretical analysis and experimental results [5, 11], fuzzy entropy performed an improved evaluation of signal complexity and has been powerfully applied to short time series contaminated by noise.

Physiological signals, such as electroencephalogram (EEG), usually exhibit complex fluctuations, uncertain disturbance and high levels of nonlinearity and non-stationarity, they also contain lots of dynamics information [12]. Investigating dynamic complexity using the notion of entropy helps us to understand complex systems better [5] and has the potential for application in clinical solutions [13]. The existence of superimposed trends in physiological signals generated by the human brain is so common that it is almost unavoidable [14]. Patients with Alzheimer's disease have been observed slowing of the EEG, reduced complexity of the EEG signals, and perturbations in EEG synchrony [15]. These findings suggested that dynamic complexity could be a potential bio-signature used to monitor a person's health condition.

With EEG signals, the high nonlinear and non-stationary brainwaves, especially superimposed trends in signals, could influence the estimation of entropy-based analysis by increasing the standard deviation of the data. Thus, in order to eliminate trend oscillations, the inherent functions (called intrinsic mode functions or IMFs) extracted from the empirical 
mode decomposition (EMD) are considered an effective filter for reducing superimposed trends in signals [16].

Therefore, it is for the above reasons that we considered EMD-based fuzzy entropy and its multiscale version for EEG study over other complexity analysis techniques. This paper proposes an inherent fuzzy entropy (Inherent FuzzyEn) algorithm and its multiscale version (Multiscale Inherent FuzzyEn or MIFE). An important benefit of Inherent FuzzyEn is the ability to adapt to EEG signals in time sequences. Since EEG complexity can distinguish patients and health controls [13], the proposed Inherent FuzzyEn algorithm is promising for its application to the healthcare solutions in the real world.

\section{ALGORITHM OF INHERENT FUZZY ENTROPY}

In this section, the algorithm of Inherent Fuzzy Entropy was represented as a flowchart (Fig. 1), and three components of Inherent Fuzzy Entropy algorithm are described in the following parts: A. EMD technology for de-trending process; B. FuzzyEn algorithm for complexity evaluation; and C. Multiscale procedure.

\section{A. EMD Technique for De-trending Process}

We apply the EMD technique to decompose the original signal $x(t)$ into several IMFs, and re-construct the signal $\hat{x}(t)$. The procedures of EMD were showed as follows.

1) Find all extrema of signal $x(t)$ :

$F_{\text {minima }}$ and $F_{\text {maxima. }}$. Interpolate between $F_{\text {minima }}$ and $F_{\text {maxima }}$, ending up with some envelope $e_{\min }(t)$ and $e_{\max }(t)$.

2) Compute the mean:

$$
m(t)=\left(e_{\min }(t)+e_{\max }(t)\right) / 2
$$

3) Extract the candidate of inherent functions:

$$
\text { IMFs is } d(t)=x(t)-m(t)
$$

4) Is $d(t)$ an IMF? $-d(t)$ is an IMF that satisfies two conditions: first, in the whole data set, the number of extrema and the number of zero crossings must either equal or differ at most by one. Second, at any point, the mean value of the envelope defined by the local maxima and the envelope defined by the local minima is zero.

If yes, save $d(t)$ and compute the residue

$$
r(t)=x(t)-\sum_{i=1}^{t} d(t)
$$

5) Do $t=t+1$ and treat $r(t+1)$ as input data. Otherwise, treat $d(t+1)$ as input data. Iterate on the residual $m(t)$. Continue until the final residue $r$ satisfies stopping criterion: when the residue, $r$, becomes a monotonic function from which no more IMF can be extracted.

6) The IMFs components surviving high trends were automatically removed by a trend filtering algorithm [17]. The remained IMFs were chosen for reconstructing signal $\widehat{x}(t)$ by cumulative sums of the remained IMFs:

$$
\hat{x}(t)=\sum_{i=n}^{i=m} d(t)
$$

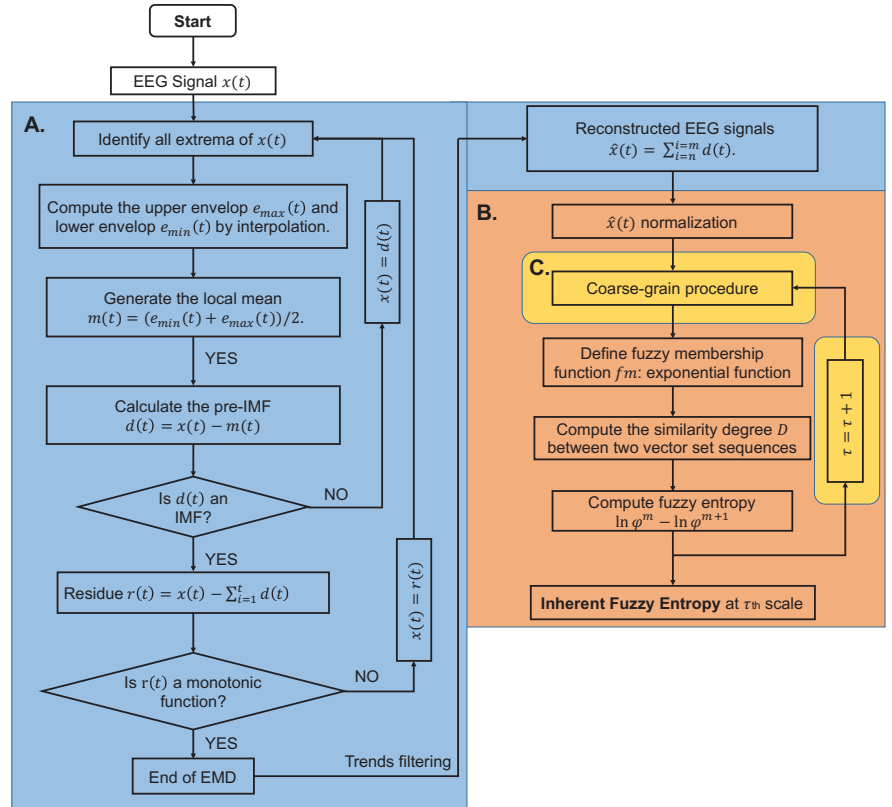

Fig. 1 Flowchart of the Inherent Fuzzy Entropy algorithm: A. EMD; B. FuzzyEn; C. Multiscale procedure.

For the parameter in Equation (3) and (4), parameter $i$ is the order number of IMFs components, and parameters $m$ and $n$ are the upper and lower boundary number of selected IMFs components, respectively.

\section{B. FuzzyEn Algorithm for Complexity Evaluation}

1) Normalize the EEG signal:

$$
y(t)^{*}=\frac{y(t)-\text { mean }(y(t))}{\text { standard deviation }}
$$

2) The FuzzyEn considering the $N$ sample time series $\{u(i): 1 \leq i \leq N\}$, given $m, n$, and $r$, and a vector set sequences $\left\{X_{i}^{m}, i=1, \ldots N-m+1\right\}$ is calculated as follows:

$X_{i}^{m}=\{u(i), u(i+1), \ldots, u(i+m-1)\}-u 0(i)$

Where $1 \leq i \leq N-m+1$, and $X_{i}^{m}$ presents $m$ consecutive $u$ values, commencing with the $i$ th point and generalized by removing a baseline:

$$
u 0(i)=m^{-1} \sum_{j=0}^{m-1} u(i+j)
$$

3) Given a vector $X_{i}^{m}$, define the similarity degree $D_{i j}^{m}$ between $X_{i}^{m}$ and $X_{j}^{m}$ by a fuzzy membership function:

$$
D_{i j}^{m}=f m\left(d_{i j}^{m}, n, r\right)
$$

Where fuzzy membership function is an exponential function:

$$
f m\left(d_{i j}^{m}, n, r\right)=\exp \left(-\frac{\left(d_{i j}^{m}\right)^{n}}{r}\right)
$$

and $d_{i j}^{m}$ is the maximum absolute difference of the corresponding scalar components of $X_{i}^{m}$ and $X_{j}^{m}$.

4) Construct the function $\varphi^{m}$ as 


$$
\varphi^{m}(n, r)=(N-m)^{-1} \sum_{i=1}^{N-m}\left((N-m-1)^{-1} \sum_{j=1, j \neq i}^{N-m} D_{i j}^{m}\right)
$$

Similarly, for $m+1$, repeat the above steps:

$$
\varphi^{m+1}(n, r)=(N-m)^{-1} \sum_{i=1}^{N-m}\left((N-m-1)^{-1} \sum_{j=1, j \neq i}^{N-m} D_{i j}^{m+1}\right)
$$

If the length of datasets $N$ is finite, the parameter $\operatorname{FuzzyEn}(m, n, r, N)$ of the sequence $\{u(i): 1 \leq i \leq N\}$ is defined as the negative natural logarithm of the deviation of $\varphi^{m}$ from $\varphi^{m+1}$ :

$$
\operatorname{FuzzyEn}(m, n, r, N)=\ln \varphi^{m}(n, r)-\ln \varphi^{m+1}(n, r)
$$

For the parameter choices of FuzzyEn, the first parameter $m$, as in ApEn and SampEn, is the length of sequences to be compared. The other two parameters $r$ and $n$ determine the width and the gradient of the boundary of the fuzzy membership function, respectively.

\section{Multiscale Version}

Multiscale version considered coarse-graining the signals into different time scales. For a given time series, multiple coarse-grained time series are constructed by averaging the data points within non-overlapping windows of increasing length, $\tau$ element of the coarse-grained time series, $y_{j}^{(\tau)}$ is calculated according to the Equation:

$$
y_{j}^{(\tau)}=\frac{1}{\tau \sum_{i=(j-1) \tau+1}^{j \tau} x_{i}}
$$

where $\tau$ represents the scale factor and $1 \leq j \leq N / \tau$. The length of each coarse-grained time series is $N / \tau$. For scale 1 , the coarse-grained time series is simply the original time series. Choosing the appropriate scales (e.g., $\tau=10$ ) before calculating FuzzyEn algorithm (Section II. B.), multiscale Inherent FuzzyEn was performed in the Fig. 1C.

\section{EXPERIMENTAL ANALYSIS}

\section{A. EEG Data Collection}

Twelve healthy young adults ( 5 men and 7 women, mean \pm std age: $31.5 \pm 2.3$ years) participated in the resting-state experiment. It was requested that all the participants had no history of neurological, psychiatric, or addictive disorders according to self-reports. No participant had taken antipsychotic or other relevant psychoactive drugs in the two preceding weeks. This study was approved by the Institutional Review Board of the Veterans General Hospital, Taipei, Taiwan. All the participants were asked to read and sign an informed consent form before participating in the EEG experiment.

In the first 5 minutes, subjects were instructed to take several deep breathings to adapt to the environment. Then subjects were instructed to open their eyes for 1 minute and close their eyes for 1 minute three times (epochs) in total (as shown in Fig. 2), meanwhile EEG signals were recorded using $\mathrm{Ag} / \mathrm{AgCl}$ electrodes by a 32-channel Quik-Cap (Compumedical

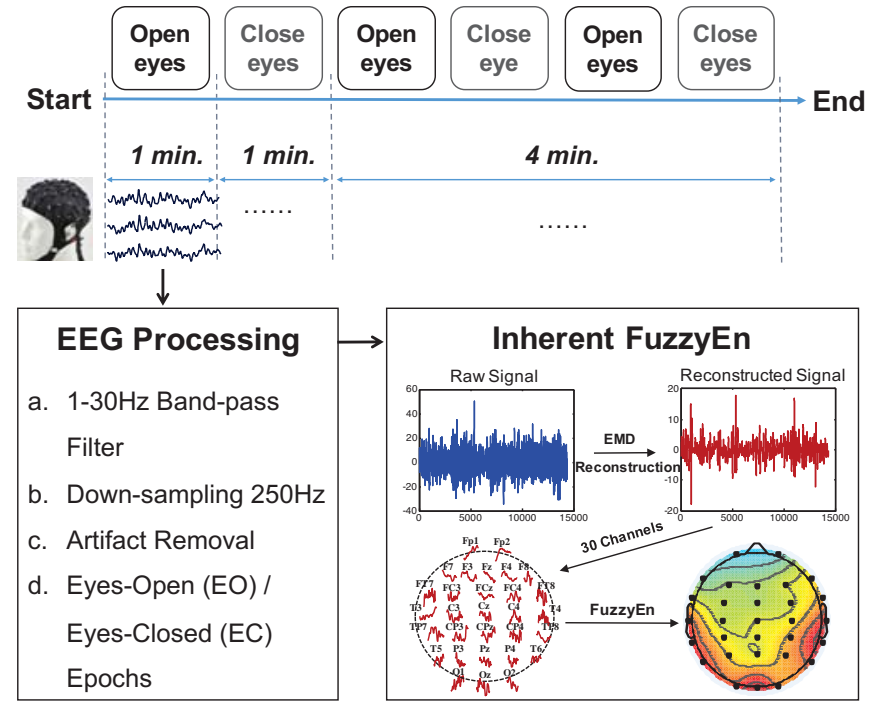

Figure 2. The analytical procedures: EEG recording, EEG processing and entropy evaluation.

NeuroScan). Thirty electrodes (Fp1, Fp2, F7, F3, Fz, F4, F8, FT7, FC3, FCz, FC4, FT8, T3, C3, Cz, C4, TP7, CP3, CPz, CP4, TP8, T5, P3, Pz, P4, T6, O1, Oz and O2) were arranged according to a modified international 10-20 system, and two reference electrodes (A1 and A2) were placed on both mastoid bones. The skin under the reference electrodes were abraded using Nuprep (Weaver and Co., USA) and disinfected with a $70 \%$ isopropyl alcohol swab before calibration. The impedance of the electrodes was calibrated under $5 \mathrm{k} \Omega$ using NaCl-based conductive gel (Quik-Gel, Neuromedical Supplies $\left.{ }^{\circledR}\right)$. The EEG signals from the electro-cap were amplified using the Scan NuAmps Express system (Compumedics Ltd., VIC, Australia) and recorded at a sampling rate of $500 \mathrm{~Hz}$ with 16 bit quantization.

\section{B. EEG Processing}

The general scheme of the EEG analysis is illustrated in Fig. 2. The acquired EEG data were processed and analyzed using EEGLAB (http://www.sccn.ucsd.edu/eeglab/, an open-source EEG toolbox for MATLAB) during the EEG processing and complexity calculation steps. For the part of EEG processing, the raw EEG signals were subjected to a 1-Hz high-pass and 30$\mathrm{Hz}$ low-pass infinite impulse response filter, and then downsampled to $250 \mathrm{~Hz}$ from the sample recording rate of $500 \mathrm{~Hz}$. For the artifact rejection, apparent eye contaminations in EEG signals were manually removed by visual inspection. Then, Independent Component Analysis (ICA) was applied to the EEG signals and the components responsible for the eye movements and blinks were rejected. Finally, the EEG signals without these artifact components was reconstructed using the back-projection method. The EEG data were segmented into eyes-open (EO) and eyes-closed (EC) epochs for further complexity analysis. The EEG complexity of EO and EC conditions were calculated and compared by the entropy evaluation (ApEn, SampEn, FuzzyEn and Inherent FuzzyEn). 


\section{RMSD Evaluation}

Dynamic complexity was evaluated by comparing 4 types of entropy-based algorithms (Inherent FuzzyEn, FuzzyEn, SampEn and ApEn) using root mean square deviation (RMSD) in EO and EC conditions. The formula of RMSD was shown as follows:

$$
R M S D=\sqrt{\left.\frac{1}{n} \sum_{i=1}^{n}\left(y_{i}-\widehat{y}_{l}\right)\right)^{2}}
$$

where $\hat{y}$ is the mean value of entropy among 3 EO or EC epochs, and $n=3$.

\section{EXPERIMENTAL RESULTS}

\section{A. EEG Complexity}

In our study, the EEG complexity of 12 participants between eyes-open (EO) and eyes-closed (EC) conditions were compared using ApEn, SamEn, FuzzyEn, and Inherent FuzzyEn algorithms (parameters $m=2, r=0.15$ and $\tau=10$ ). As shown in the Fig. 3, the EEG complexity of each epoch (EO1, EC1, EO2, EC2, EO3, and EC3) was the averaged entropy value from all participants.

By comparison of EEG complexity between EO and EC conditions, our results showed that the Inherent FuzzyEn employs the higher effect sizes than the other shallow models (ApEn, SamEn and FuzzyEn). Specifically, the Inherent FuzzyEn performed the significantly higher parietal and occipital and lower frontal EEG complexity in the EO condition (False Discovery Rate-adjusted $\mathrm{p}<.05$ ), relative to that in the EC condition (Statistics: Wilcoxon signed-rank test). Moreover, fuzzy-based entropy models have more distinguished EEG complexity (EO vs. EC) than non-fuzzy entropy models. Furthermore, the Inherent FuzzyEn demonstrated less variation than FuzzyEn, SampEn and ApEn in $3 \mathrm{EO}$ epochs.

\section{B. RMSD Evaluation}

The feasibility of evaluating dynamic complexity in EO and EC conditions was examined by comparing ApEn, SampEn, FuzzyEn and Inherent FuzzyEn using RMSD. The performances of RMSD for ApEn, SampEn, FuzzyEn and Inherent FuzzyEn based on 1-30 channels (Fp1, Fp2, F7, F3, Fz, F4, F8, FT7, FC3, FCz, FC4, FT8, T3, C3, Cz, C4, TP7, CP3, CPz, CP4, TP8, T5, P3, Pz, P4, T6, O1, Oz and O2) are described in Fig. 4. The results show that fuzzy-based entropy models have less RMSD than non-fuzzy entropy models in EO and EC conditions. Most importantly, the Inherent FuzzyEn algorithm has the lowest RMSD in all channels relative to previous entropy models in EO and EC conditions, which suggests the proposed algorithm provides the better reliability for complexity evaluation.

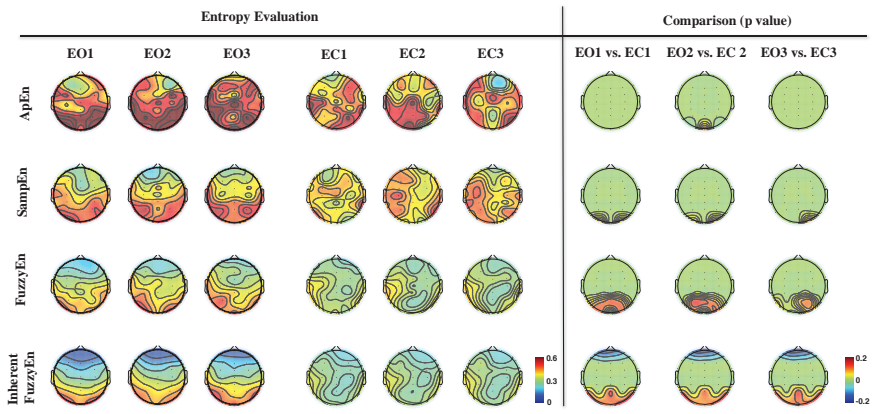

Figure 3. The EEG dynamic complexity by ApEn, SampEn, FuzzyEn and Inherent FuzzyEn evaluations.
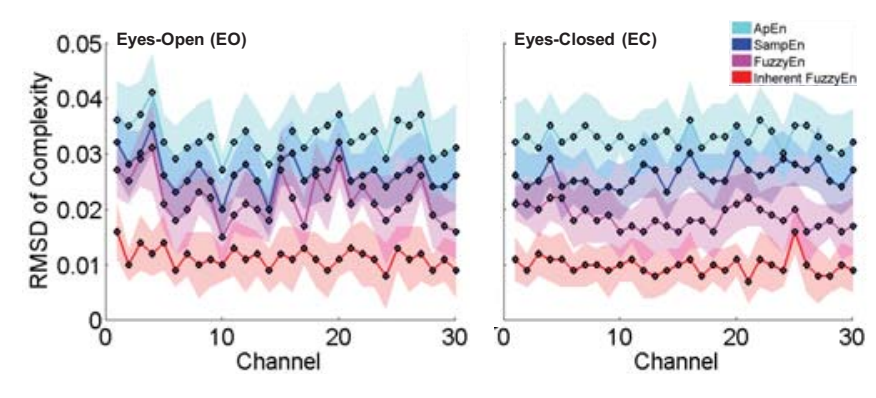

Figure 4. Complexity evaluation using RMSD in EO and EC conditions. The dash lines and envelope areas are the mean values and standard deviations, respectively.

\section{CONCLUSION}

EEG complexity is fundamentally mercurial and varying during EEG study. In previous studies, researchers have generally ignored the superimposed trends in signals. To improve performance in realistic EEG applications, using an Inherent FuzzyEn algorithm can be made more effective by collecting EEG signals from healthy subjects, which endows fuzzy membership function with EMD function. In this paper, we compared the EEG results (entropy values and RMSD) obtained using fuzzy structures (Inherent FuzzyEn and FuzzyEn) and non-fuzzy structures (SampEn and ApEn). Our findings showed that systems with fuzzy structures exhibit improved performance. Furthermore, the performance of the Inherent FuzzyEn algorithm was superior to FuzzyEn, SampEn and ApEn models. 


\section{REFERENCES}

[1] A. Humeau-Heurtier, "The multiscale entropy algorithm and its variants: A Review," Entropy, vol. 17, pp. 3110-3123, 2015.

[2] S. M. Pincus, "Approximate entropy as a measure of system complexity," Proc. Natl. Acad. Sci. USA, vol. 88, pp. 2297-2301, 1991.

[3] M. Costa, A. L. Goldberger, and C.-K. Peng, "Multiscale entropy analysis of complex physiologic time series," Phys. Rev. Lett., vol. 89, p. 068102, 2002.

[4] J. S. Richman and J. R. Moorman, "Physiological time-series analysis using approximate entropy and sample entropy," Am. J. Physiol. Heart Circ. Physiol., vol. 278, pp. H2039-H2049, 2000.

[5] W. Chen, Z. Wang, H. Xie, and W. Yu, "Characterization of surface EMG signal based on fuzzy entropy," IEEE Trans. Neural. Syst. Rehabil. Eng., vol. 15, pp. 266-272, 2007.

[6] S.-F. Liang, C.-E. Kuo, F.-Z. Shaw, Y.-H. Chen, C.-H. Hsu, and J.Y. Chen, "Combination of expert knowledge and a genetic fuzzy inference system for automatic sleep staging," IEEE Trans. Biomed. Eng., vol. 63, pp. 2108-2118, 2016.

[7] C. Yang, Z. Deng, K.-S. Choi, and S. Wang, "Takagi-Sugeno-Kang Transfer Learning Fuzzy Logic System for the Adaptive Recognition of Epileptic Electroencephalogram Signals," IEEE Trans. Fuzzy Syst., vol. 24, pp. 1079-1094, 2016.

[8] Y.-T. Liu, Y.-Y. Lin, S.-L. Wu, C.-H. Chuang, and C.-T. Lin, "Brain dynamics in predicting driving fatigue using a recurrent selfevolving fuzzy neural network," IEEE Trans. Neural Netw. Learn. Syst., vol. 27, pp. 347-360, 2016.

[9] R. Chai, S. H. Ling, G. P. Hunter, Y. Tran, and H. T. Nguyen, "Brain-computer interface classifier for wheelchair commands using neural network with fuzzy particle swarm optimization," IEEE J. Biomed. Health Inform., vol. 18, pp. 1614-1624, 2014.

[10] L. Zadeh, "Fuzzy Sets," Inf. Contro., vol. 8, pp. 338-353, 1965.

[11] W. Chen, J. Zhuang, W. Yu, and Z. Wang, "Measuring complexity using fuzzyen, apen, and sampen," Med. Eng. Phys., vol. 31, pp. 6168, 2009.

[12] M. Costa, A. L. Goldberger, and C.-K. Peng, "Multiscale entropy analysis of biological signals," Phys. Rev. E, vol. 71, p. 021906, 2005.

[13] A. C. Yang, S.-J. Wang, K.-L. Lai, C.-F. Tsai, C.-H. Yang, J.-P. Hwang, et al., "Cognitive and neuropsychiatric correlates of EEG dynamic complexity in patients with Alzheimer's disease," Prog. Neuropsychopharmacol. Biol. Psychiatry, vol. 47, pp. 52-61, 2013.

[14] K. Hu, P. C. Ivanov, Z. Chen, P. Carpena, and H. E. Stanley, "Effect of trends on detrended fluctuation analysis," Phys. Rev. E, vol. 64, p. 011114, 2001.

[15] J. Dauwels, F. Vialatte, and A. Cichocki, "Diagnosis of Alzheimer's disease from EEG signals: where are we standing?," Curr. Alzheimer. Res., vol. 7, pp. 487-505, 2010.

[16] N. E. Huang, Z. Shen, S. R. Long, M. C. Wu, H. H. Shih, Q. Zheng, et al., "The empirical mode decomposition and the Hilbert spectrum for nonlinear and non-stationary time series analysis," Proc. R. Soc. London, Ser. A, 1998, pp. 903-995.

[17] A. Moghtaderi, P. Flandrin, and P. Borgnat, "Trend filtering via empirical mode decompositions," Comput. Stat. Data Anal., vol. 58, pp. 114-126, 2013. 